\title{
Prevalência de Eventos Adversos e fatores relacionados à perda acidental de dispositivos invasivos em um Centro de Terapia Intensiva
}

\author{
Prevalence of Adverse Events and factors related to accidental loss of invasive devices in an \\ Intensive Care Unit \\ Prevalencia de eventos adversos y factores relacionados con la pérdida accidental de dispositivos \\ invasivos en una Centro de Tratamiento Intensivo
}

Recebido: 18/07/2021 | Revisado: 27/07/2021 | Aceito: 25/08/2021 | Publicado: 27/08/2021

\author{
Ruy de Almeida Barcellos \\ ORCID: https://orcid.org/0000-0002-7857-2151 \\ Hospital de Clínicas de Porto Alegre, Brasil \\ E-mail: rbarcellos@hcpa.edu.br \\ Marina Brandalise \\ ORCID: https://orcid.org/0000-0002-1692-3550 \\ Hospital de Clínicas de Porto Alegre, Brasil \\ E-mail: mbrandalise@hcpa.edu.br \\ Lucas Simeão Funck \\ ORCID: https://orcid.org/0000-0002-0783-1865 \\ Universidade Federal do Rio Grande do Sul, Brasil \\ E-mail: lucas.funck@ufrgs.br \\ Thaís dos Santos Donato Schmitz \\ ORCID: https://orcid.org/0000-0002-9608-7707 \\ Hospital de Clínicas de Porto Alegre, Brasil \\ E-mail:tschmitz@hcpa.edu.br
}

\begin{abstract}
Resumo
Objetivo: Caracterizar os eventos adversos e fatores relacionados à perda acidental de dispositivos invasivos em um centro de terapia intensiva. Metodologia: Trata-se de um estudo transversal retrospectivo. Foram utilizados dados de pacientes internados em um Centro de Tratamento Intensivo (CTI), no período de julho de 2018 a julho de 2019, que sofreram eventos adversos. Resultados: A amostra foi composta por 218 indivíduos com os quais ocorreram eventos adversos, sendo 62,8\% do sexo masculino, com idade média de 59,8 anos. Esta amostra de 218 indivíduos representou, em um total de 882 pacientes internados no CTI ao longo do mesmo período de tempo, uma prevalência de $24,72 \%$. A ocorrência de delirium esteve prevalente em 33,9\% dos casos. O tempo médio de permanência hospitalar foi de 10 dias em CTI e de 35 dias no setor de internação. Referente à distribuição dos eventos adversos, a maior ocorrência foi relacionada à perda acidental de sonda enteral $(49,1 \%)$. Destes, 156 eventos $(71,6 \%)$ foram caracterizados sem dano e $62(28,4 \%)$ como dano leve, evoluindo para recuperação sem sequelas $(99,80 \%)$. Conclusão: Salienta-se que o hospital em questão apresentou um bom indicador de qualidade no que tange os acidentes ocasionados por dispositivos invasivos. Entretanto, o aperfeiçoamento dos cuidados frente aos pacientes deve ser aprimorado constantemente, a fim de minimizar eventos adversos e fortalecer a cultura do cuidado.

Palavras-chave: Dano ao Paciente; Gestão da Segurança; Segurança do Paciente; Cuidados Críticos; Cuidados de Enfermagem.
\end{abstract}

\begin{abstract}
Objective: To characterize the adverse events related to the accidental loss of invasive devices in an intensive care unit. Methodology: This is a retrospective quantitative study. Data from patients admitted to an Intensive Care Unit (ICU), from July 2018 to July 2019, who suffered adverse events was used. Results: The sample consisted of 218 individuals with which adverse events occurred, $62.8 \%$ of those being male, with an average age of 59.8 years. This sample of 218 individuals represented, among a total of 882 patients admitted throughout the same period, a prevalence of $24,78 \%$. The occurrence of delirium was prevalent in $33.9 \%$ of cases. The average length of hospital stay was 10 days in the ICU and 35 days in the hospitalization sector. Regarding the distribution of adverse events, the highest occurrence was related to accidental loss of enteral tube (49.1\%). Of these, 156 events $(71.6 \%)$ were characterized as without damage and $62(28.4 \%)$ as mild damage, evolving to recovery without sequelae $(99.80 \%)$. Conclusion: It should be noted that the hospital in question presented a good indicator of quality about accidents caused by invasive devices. However, the improvement of care for patients must be constantly improved, in order to minimize adverse events and strengthen the culture of care.
\end{abstract}


Keywords: Patient Harm; Safety Management; Patient Safety; Critical Care; Nursing Care.

\begin{abstract}
Resumen
Objetivo: Caracterizar los eventos adversos relacionados con la pérdida accidental de dispositivos invasivos en una unidad de cuidados intensivos. Metodología: Se trata de un estudio transversal retrospectivo. Se utilizaron datos de pacientes ingresados en una Unidad de Cuidados Intensivos (UCI) de julio de 2018 a julio de 2019, que sufrieron eventos adversos. Resultados: La muestra estuvo conformada por 218 individuos con los que ocurrieron eventos adversos, 62,8\% varones, con una edad media de 59,8 años. Esta muestra de 218 individuos representó, en un total de 882 pacientes ingresados en la UCI en el mismo período de tiempo, una prevalencia del $24,72 \%$. La ocurrencia de delirio fue prevalente en el 33,9\% de los casos. La duración media de la estancia hospitalaria fue de 10 días en la UCI y de 35 días en el sector hospitalario. En cuanto a la distribución de eventos adversos, la mayor ocurrencia se relacionó con la pérdida accidental de sonda enteral $(49,1 \%)$. De estos, 156 eventos $(71,6 \%)$ se caracterizaron como sin daño y $62(28,4 \%)$ como daño leve, progresando a la recuperación sin secuelas $(99,80 \%)$. Conclusión: Cabe destacar que el hospital en cuestión presentó un indicador de buena calidad en cuanto a accidentes causados por dispositivos invasivos. Sin embargo, la mejora de la atención a los pacientes debe mejorarse constantemente para minimizar los eventos adversos y fortalecer la cultura de la atención.
\end{abstract}

Palabras clave: Daño del paciente; Administración de la Seguridad; Seguridad del paciente; Cuidado Críticos; Atención de Enfermería.

\title{
1. Introdução
}

Anualmente, acontecem cerca de 42,7 milhões de incidentes em internações hospitalares (Françolin et al., 2015). Um estudo prospectivo brasileiro constatou a ocorrência de 9,3 eventos adversos por 100 pacientes-dia, resultando em um aumento de 19 dias no tempo de internação hospitalar, duplicando, assim, a chance de óbito (Roque et al., 2016).

Os incidentes hospitalares podem ser classificados da seguinte forma: (a) circunstância notificável, quando há um incidente com potencial dano ou lesão; (b) Near miss, quando o incidente não atinge o paciente; (c) Incidente sem dano, quando o incidente atinge o paciente, mas não causa dano; (d) Incidente com dano, também conhecido como evento adverso, quando o incidente atinge o paciente e resulta em lesão ou dano (Sousa \& Mendes, 2019).

Compreende-se por incidente com dano ou evento adverso a intercorrência não intencional ocasionado ao paciente, proveniente das intervenções da equipe de saúde, que podem gerar um dano ou lesão que acarrete desconforto físico e emocional, incapacidade, aumento no tempo de internação hospitalar e óbito (World Health Organization, 2010).

Em 2004, a Organização Mundial da Saúde (OMS) propulsionou a discussão referente às questões de segurança na área da saúde e estimulou a criação da Aliança Mundial para a segurança do paciente. Em 2008, foi criada a Rede Brasileira de Segurança do Paciente no Brasil, visando ao cuidado seguro e à qualidade da assistência. Contudo, somente em 2013, através da portaria n 529 do Ministério da Saúde (MS), o tema ganhou ênfase com o Programa Nacional de Segurança do Paciente (PNSP) instituindo estratégias de implementação para todas as instituições nacionais de saúde (Alves et al., 2016; Minuzzi et al., 2016).

Sabe-se que os pacientes graves, que apresentam quadro instável e que são submetidos a diversas intervenções clínicas, a fim de obter o diagnóstico ou de desenvolver a sua reabilitação, possuem maior risco para a ocorrência de eventos adversos. Por isso, o cuidado oferecido ao paciente crítico é complexo e requer equipamentos de alta performance tecnológica e de profissionais especializados, que sejam competentes em intervir de forma imediata em qualquer alteração do quadro clínico a que o paciente venha a se encontrar (Roque et al., 2016).

Em instituições hospitalares, pacientes internados em Unidades de Terapia Intensiva (UTI) possuem maior vulnerabilidade de sofrerem erros assistenciais ou eventos adversos em decorrência da demanda e complexidade de cuidados, visto que possuem instabilidade hemodinâmica e gravidade clínica (Novaretti et al., 2014; Oliveira et al., 2016). Dentre os eventos adversos em terapia intensiva, evidencia-se maior prevalência relacionada a terapia medicamentosa (65\%), seguido de infecções relacionadas à assistência de saúde (IRAS), quedas, lesão por pressão, flebites e retirada acidental de drenos e 
sondas, bem como extubação (Lorenzini et al., 2014; Nunes et al., 2014).

Este estudo se justifica pela preocupação com a qualidade da assistência oferecida ao paciente e com a minimização dos riscos assistenciais. O gerenciamento de riscos pode auxiliar na redução de eventos ao paciente, pois proporcionam aos gestores informações importantes, que contribuam na tomada de decisões na instituição, para que ações sejam priorizadas, a fim de mitigar os riscos.

Constata-se que a enfermagem desempenha papel fundamental no gerenciamento de risco, uma vez que permanece a maior parte do tempo com a paciente e pode detectar previamente potenciais riscos e estabelecer medidas preventivas para que as situações de risco não se tornem eventos adversos (Fassini \& Hahn, 2012). Isto é, através do cuidado oferecido por profissionais capacitados da área da enfermagem, pode-se reconhecer, gerenciar e prevenir riscos (Barcellos et al., 2016).

Pensando nisso, as instituições de saúde têm prezado pela excelência da assistência prestada com ênfase na segurança do paciente, no intuito de minimizar complicações clínicas graves e óbitos decorrentes de eventos adversos, os quais poderiam ser evitáveis (Alves et al., 2016).

Assim, tendo em vista a necessidade e a importância de qualificar a assistência prestada no âmbito da saúde, de prevenir eventos adversos e de promover a segurança do paciente, este estudo objetiva caracterizar os eventos adversos e fatores relacionados à perda acidental de dispositivos invasivos em um centro de terapia intensiva.

\section{Metodologia}

Trata-se de um estudo transversal retrospectivo, realizado em um Centro de Terapia Intensiva (CTI) de um hospital de grande porte da capital do estado do Rio Grande do Sul.

Foram incluídos no estudo 218 pacientes que tiveram eventos adversos relacionados à perda acidental de dispositivos invasivos notificados, admitidos no CTI da instituição participante no período de julho de 2018 a julho de 2019. Foram excluídos das análises os pacientes que não tiveram a análise do evento finalizada até o término do período de coleta de dados.

A coleta de dados foi realizada retrospectivamente em prontuário eletrônico, a partir de informações relativas aos

dados sociodemográficos e variáveis clínicas, que pudessem ter relação com o desfecho. As variáveis clínicas analisadas foram: 1) sexo, 2) idade, 3) prevalência de delirium, 4) dor, 3) avaliação conforme escala SAPS 3, 4) necessidade de contenção mecânica e 5) tempo de permanência no CTI.

O sistema prognóstico Simplified Acute Physiology Score 3 (SAPS-3) foi utilizado para estabelecimento de um índice preditivo de mortalidade através do escore obtido com base em 20 variáveis, representadas por escore fisiológico agudo e avaliação do estado prévio.

Os dados foram inseridos em planilha Microsoft Excel (2015) e posteriormente transferidos para o Software Statistical Package for Social Sciences (SPSS) versão 21.0 para a análise estatística.

Realizou-se análise descritiva, os dados categóricos foram apresentados por frequências absolutas e relativas. A descrição das variáveis contínuas foi apresentada através de média e desvio padrão ou mediana e intervalo interquartil, conforme a simetria das variáveis.

O projeto de pesquisa foi aprovado pela Comissão Científica e Comitê de Ética em Pesquisa do Hospital de Clínicas de Porto Alegre sob o número 2.607.821. Por se tratar de pesquisa utilizando dados secundários, dispensou-se o uso de Termo de Consentimento Livre e Esclarecido, o qual foi substituído pelo Termo de utilização de banco de dados.

\section{Resultados e Discussão}


A amostra foi composta por 218 indivíduos que sofreram incidentes relacionados à perda acidental de dispositivos, de um total de 882 pacientes internados no CTI ao longo do período do estudo, uma prevalência de 24,72\%. Dos pacientes que sofreram incidentes, 137 (62,8\%) eram do sexo masculino, com idade média de 59,8 anos. Foi observado prevalência de delirium em 74 pacientes $(33,9 \%)$ e de dor em 68 pacientes (31,2\%). A pontuação média obtida para o escore SAPS 3 foi de 62,7 .

O tempo de permanência hospitalar da população analisada foi de dez dias em CTI e de 35 dias em setor de internação conforme indica a Tabela 1 .

Tabela 1. Caracterização geral da amostra. Porto Alegre, 2018-2019.

\begin{tabular}{|c|c|c|c|c|}
\hline Variáveis & & & $n=218$ & $\%$ \\
\hline \multicolumn{5}{|l|}{ Sexo } \\
\hline Feminino & & & 81 & 37,2 \\
\hline Masculino & & & 137 & 62,8 \\
\hline Idade Média \pm DP & & & $59,8 \pm 16,4$ & \\
\hline Delirium & & & 74 & 33,9 \\
\hline Dor & & & 68 & 31,2 \\
\hline SAPS 3 Média \pm DP & & & $62,7 \pm 17,6$ & \\
\hline Contenção mecânica & & & 70 & 32,1 \\
\hline Dias permanência & - mediana (p25 & $-p 75)$ & & \\
\hline CTI & & & $10(1-68)$ & \\
\hline Hospitalar & & & $35(4-290)$ & \\
\hline
\end{tabular}

Fonte: Dados da pesquisa.

A maior ocorrência de incidentes foi relacionada à perda acidental de sonda enteral, a qual foi detectada em 107 pacientes $(49,1 \%)$, durante o turno da noite $(90-41,3 \%)$, sendo a equipe de enfermagem a principal notificadora $(211$ 96,8\%). Destes, 156 incidentes (71,6\%) não causaram danos aos pacientes e 62 eventos (28,4\%) acarretaram dano leve, evoluindo para recuperação sem sequelas 216 destes $(99,8 \%)$, conforme ilustrado na Tabela 2. 
Tabela 2. Distribuição dos eventos adversos. Porto Alegre, 2018-2019.

\begin{tabular}{|c|c|c|}
\hline Variáveis & $\mathrm{n}=\mathbf{2 1 8}$ & $\%$ \\
\hline \multicolumn{3}{|l|}{ Perda Acidental } \\
\hline Sonda enteral & 107 & 49,1 \\
\hline Cateteres venosos e arteriais & 56 & 25,7 \\
\hline Sonda vesical & 9 & 4.1 \\
\hline Drenos & 8 & 3,7 \\
\hline Outros* & 38 & 17,4 \\
\hline \multicolumn{3}{|l|}{ Turno } \\
\hline Manhã & 72 & 33 \\
\hline Tarde & 56 & 25,7 \\
\hline Noite & 90 & 41,3 \\
\hline \multicolumn{3}{|l|}{ Notificador } \\
\hline Equipe de Enfermagem & 211 & 96,8 \\
\hline Equipe Médica & 4 & 1,8 \\
\hline Outros & 3 & 1,4 \\
\hline \multicolumn{3}{|l|}{ Gravidade Do Dano } \\
\hline Sem dano & 156 & 71,6 \\
\hline Dano leve & 62 & 28,4 \\
\hline \multicolumn{3}{|l|}{ Evolução Do Evento } \\
\hline Recuperado sem sequelas & 216 & 99,8 \\
\hline Recuperado com sequelas & 2 & 0,91 \\
\hline
\end{tabular}

*Outros (Picc, acesso venoso periférico, cateter peridural), etc..

Fonte: Dados da pesquisa.

Há evidências de que a maioria dos eventos adversos relacionados à perda acidental de dispositivos ocorrem em pessoas que se encontram na sexta década de vida (Brandão et al., 2018; Roque et al., 2016). A literatura indica que os idosos alocados na UTI são provenientes, em sua maioria, dos serviços de urgência, em consequência das alterações clínicas, e permanecem um longo período na unidade, de forma que são os protagonistas quando se trata de eventos adversos (Toffoletto et al., 2016).

Diversos são os pacientes internados na UTI que manifestam o distúrbio de delirium. A condição diz respeito a uma disfunção neurológica aguda e pode ser observado em pacientes internados nas UTIs, em função das alterações transitórias da consciência e cognição, geralmente são detectados em um curto período de tempo. Os pacientes que possuem essa comorbidade não apresentam evolução significativa, o que contribui com o aumento do tempo de internação hospitalar, maior ocorrência de eventos adversos e possibilidade de morte (Dessap et al., 2015).

A prevalência de delirium constatada - 33,9\% - é compatível com outros dados presentes na literatura latinoamericana. Lago et al (2020), em estudo com 59 pacientes, constataram prevalência idêntica de 33,9\%. Outro estudo com 335 pacientes observou uma prevalência da síndrome de 36\% (Martins et al., 2019), enquanto Matioli et al. (2021) constataram prevalência de $40 \%$ entre 50 pacientes avaliados. Salienta-se, no entanto, que outros estudos realizados apresentam dados 
significativamente distintos em relação à prevalência de delirium, seja essa menor como constatado por Barcellos et al. (2020) - equivalente a $8 \%$ em 1271 pacientes, ou maior - 80\% em 230 pacientes (Mesa et al., 2017).

Acrescenta-se que alguns medicamentos utilizados em terapia intensiva, como propofol, morfina e anticolinérgicos também auxiliam a potencializar o desenvolvimento do delirium. Alguns dos propósitos da sedação consistem em tornar o paciente cooperativo e calmo, com sono apropriado, sem dor e minimamente ansioso. Isto é, na maioria dos casos, para que ele não sinta dor, é necessário medicá-lo, o que faz com que ele delire (Dessap et al., 2015).

Em estudo realizado com 355 pacientes, identificou-se que o tempo médio de permanência no CTI de pacientes com evento adverso é de aproximadamente 8,9 dias. Identificou-se também significativa relação entre o evento adverso e a mortalidade, de forma que quanto mais tempo o paciente permanece na unidade de terapia intensiva maior é a taxa óbitos. Isso demonstra a gravidade dos problemas ocasionados por danos associados ao cuidado em saúde e a necessidade de desenvolvimento de ações direcionadas ao processo assistencial e a redução de incidentes (Roque et al., 2016).

O tempo de permanência em UTI de 10 dias entre os 218 pacientes objetos deste estudo mostrou-se acima da média do total de internações em UTI na mesma instituição durante o mesmo período de tempo - 7 dias. Este valor varia de acordo com as características e as necessidades do hospital e de cada paciente. Pode-se dizer, que permanências mais curtas do que o esperado indicam que diversos esforços foram desempenhados para diminuir custos hospitalares ou que, em função da gravidade dos casos, houve morte nos primeiros dias e horas da internação. Em contrapartida, tempos de permanência mais longos podem relacionar-se com baixa qualidade no atendimento prestado, uma vez que esse tempo pode ser resultado de complicações resultantes do cuidado deficitário e até mesmo em função dos eventos adversos (Ortega et al., 2017).

Resultados semelhantes foram encontrados em outros estudos, os quais identificaram a perda acidental da sonda enteral como o evento de maior prevalência (Barcellos et al., 2016; Lima \& Barbosa, 2015). Sabe-se que a perda de tubos, sondas e cateteres é frequente nas unidades de terapia intensiva, a qual condiz com a expulsão deliberada desses dispositivos pelo próprio paciente e até mesmo pelos cuidados prestados pela equipe multiprofissional. Evidencia-se que os fatores relacionados à perda da sonda de alimentação são inúmeros e implicam diretamente na desnutrição iatrogênica e necessidade de reinstalação do dispositivo para a alimentação, o que causa desconforto ao paciente e a maiores custos relacionados a materiais e procedimento (Souza et al., 2018).

Diversas são as causas da perda da sonda enteral e elas podem estar diretamente relacionadas com a obstrução do lúmen interno por incrustação de medicamentos e/ou dietas, fixação ineficaz com perda parcial ou total desta, procedimentos médicos e de enfermagem, que condicionam a tração e a necessidade de reposicionamento, êmese e tosse que propiciam à expulsão acidental, além da ausência de registros do motivo da perda, entre outros fatores (Souza et al., 2018).

No que concerne à equipe notificadora dos eventos adversos, salienta-se que, geralmente, os técnicos de enfermagem relatam aos enfermeiros (ou ao enfermeiro responsável) da unidade, os quais repassam ao médico assistente do paciente, conforme a situação, sem omitir informações. Todas as ocorrências devem ser inseridas no prontuário eletrônico do paciente. Enfatiza-se que o enfermeiro é o profissional responsável pela liderança da equipe de enfermagem, constituída principalmente por profissionais do nível técnico. Entre as atividades realizadas por ele está a supervisão e orientação da equipe diante das situações cotidianas, bem como as orientações para a execução das tarefas (Fassini \& Hahn, 2012). Desta forma, provavelmente a equipe de enfermagem, por estar mais próxima do paciente durante procedimentos e rotinas diárias e por conhecer melhor os processos de gerenciamento de risco, seja a responsável pelo maior número de notificações dos eventos adversos.

Ao levarmos em consideração o escore médio obtido através da aplicação da ferramenta prognóstica Simplified Acute Physiology Score 3 (SAPS-3) neste estudo (62,7) estima-se uma taxa de mortalidade na população analisada de 39,8\%. O sistema SAPS-3, elaborado em 2002 em um estudo de coorte de escala mundial, avalia 20 diferentes variáveis no momento da 
admissão do paciente, sendo estas divididas em três partes: variáveis demográficas, fisiológicas e variáveis relacionadas ao motivo da internação na UTI, sendo atribuído a cada uma destas um peso correspondente à sua gravidade. O escore obtido, podendo este variar entre 16 a 217 pontos, visa estimar a mortalidade do indivíduo avaliado. Estudos realizados em UTIs de hospitais do Brasil apontam uma boa aplicabilidade e especificidade do sistema SAPS-3 na população brasileira, destacando a sua utilidade na indicação de pacientes graves e subsequente organização dos cuidados necessários (Bueno et al., 2015; De Assis et al., 2020; Leão et al., 2019; Silva Junior et al., 2010).

Sabe-se da importância do entendimento e da identificação das implicações dos eventos adversos no âmbito hospitalar para prevenir tais riscos e impedir que situações como essa ocorram. Bellaguarda et. al. (2020) argumentam a favor da construção de bundles de prevenção como estratégia para o aprimoramento de processos assistenciais e a promoção de um cuidado mais seguro, baseando-se no conceito da prática baseada em evidências. Estes bundles, ou pacotes, são compostos por um pequeno número de intervenções recomendadas em relação a um processo de cuidado específico, que quando utilizadas de maneira correta, coletiva e com boa adesão pela equipe, promovem uma melhora deste cuidado para o paciente. Tais benefícios da implementação de bundles de cuidados na assistência em saúde são já bem evidenciados, sendo eles de simples elaboração e fácil aplicação, além de terem baixos custos financeiros e um baixo impacto na carga de trabalho da equipe aderente (Chicayban et al., 2017; Coelho et al., 2020; Colaço \& Nascimento, 2014; Oliveira \& Nunes, 2015). No entanto, destaca-se que a eficácia da implementação de um determinado bundle está fortemente associada ao treinamento periódico da equipe acerca do conteúdo nele incluso, a fim de estimular o nível de adesão necessário para que este tenha o impacto desejado como indicador de qualidade no cuidado (Llapa-Rodríguez et al., 2019).

\section{Considerações Finais}

A ocorrência do evento adverso implica em uma grave problemática na assistência à saúde no âmbito da terapia intensiva, impactando no aumento do tempo de internação hospitalar e mortalidade. Entre os diversos dispositivos invasivos identificados, a predominância da perda da sonda enteral é uma tendência presente na literatura, dado que enfatiza a importância da elaboração de medidas de cunho preventivo em relação à perda destes dispositivos.

Entende-se que prevalência de delirium constatada no presente estudo, similar a outros dados disponíveis na literatura, deve mesmo assim ser considerada alta. Visto o impacto significativo que o distúrbio suscita no quadro do paciente, suas possíveis complicações e o subsequente aumento da carga de trabalho da equipe de saúde para manejá-las, julga-se vantajoso o incentivo a práticas e rotinas preventivas em relação à síndrome, assim como a promoção de estudos que visem aprimorá-las.

Nota-se uma expansão relevante à cerca da ocorrência de eventos adversos relacionados à assistência em saúde, em parte devido à ascensão de cuidados com a segurança do paciente. Os resultados encontrados neste estudo reforçam a importância da disseminação de uma cultura de segurança no ambiente hospitalar, de suma importância em áreas de elevada complexidade como as UTIs.

O papel da equipe de enfermagem no que diz respeito ao reconhecimento e notificação de eventos adversos relacionados à perda de dispositivos invasivos é salientado através nos dados obtidos, evidenciando a importância do seu preparo e conhecimento para que possam lidar de forma ágil e correta frente a estes eventos. Entende-se então que o investimento em práticas e ações que visem desenvolver e aprimorar estas suas capacidades - tanto interventivas quanto preventivas - seria de grande benefício na prestação de cuidado de maior qualidade ao paciente.

A elaboração de novas pesquisas com uma temática preventiva deve ser incentivada, com estudos controlados que avaliem associações às diversas variáveis clínicas, favorecendo assim o monitoramento do desfecho com a finalidade de subsidiar ações de redução de eventos adversos, bem como qualificar de forma fundamentada a assistência a ser prestada. 
Julga-se imprescindível o investimento por parte da instituição de saúde no desenvolvimento individual dos integrantes de suas equipes de saúde. Distanciando-se de qualquer raciocínio punitivo, é necessário investir no aprimoramento da sua capacidade de reconhecer potenciais riscos, tal que diferentes maneiras de eliminar ou reduzi-los possam ser empregadas.

\section{Referências}

Alves, K. Y. A., Costa, T. D. da, Barros, A. G. de, Lima, K. Y. N. de, \& Santos, V. E. P. (2016). Patient safety in intravenous therapy in the intensive care unit. Revista de Pesquisa: Cuidado é Fundamental Online, 8(1), 3714.

Barcellos, R. de A., Toscan, M., Báo, A. C. P., \& Amestroy, S. C. (2016). Effectiveness of Clinical Risk Management in Intensive Care. Cogitare Enferm, 21, $1-8$.

Barcellos, R. de A., Zanon, A., Castilhos, T. de C., Candaten, A. E., \& Báo, A. C. P. (2020). Prevalência de delirium em terapia intensiva adulto. Research, Society and Development, 9(8), e165985431. https://doi.org/10.33448/rsd-v9i8.5431

Bellaguarda, M. L. dos R., Vieira, I. M. S., Petri, J. H., Coelho, R., \& Moraes, C. L. K. (2020). Bundle de prevenção das complicações da sondagem nasoenteral em unidade de terapia intensiva. Global Academic Nursing Journal, 1(2), 1-7. https://doi.org/10.5935/2675-5602.20200018

Brandão, M. G. S. A., Brito, O. D., \& Barros, L. M. (2018). Gestão de riscos e segurança do paciente: mapeamento dos riscos de eventos adversos na emergência de um hospital de ensino. Revista de Administração Em Saúde, 18(70).

Bueno, H. L., Francisco, J., \& Biatto, P. (2015). Epidemiologia E Validação De Escore Prognóstico Em Uti Mista Do Norte Do Paraná Epidemiology and Validation of Prognosys Scores in Intensive Care Unit of North of Parana State. 22(3), 23-29. http://www.mastereditora.com.br/review

Chicayban, L. M., Souza Terra, É. L. V., Ribela, J. dos S., \& Barbosa, P. F. (2017). Bundles De Prevenção De Pneumonia Associada À Ventilação Mecânica: a Importância Da Multidisciplinaridade. Biológicas \& Saúde, 7(25), 25-35. https://doi.org/10.25242/886872520171200

Coelho, D. F., Demarche, N. F., Ficagna, F. T., \& Valcarenghi, R. V. (2020). O Impacto da utilização de Bundles na prevenção da pneumonia associada à ventilação mecânica. Revista Enfermagem Atual In Derme, 92(30), 6-20. https://doi.org/10.31011/reaid-2020-v.92-n.30-art.468

Colaço, A. D., \& Nascimento, E. R. P. do. (2014). Nursing intervention bundle for enteral nutrition in intensive care: A collective construction. Revista Da Escola de Enfermagem, 48(5), 841-847. https://doi.org/10.1590/S0080-623420140000500010

De Assis, L. G. R., Nery Neto, C. D. S., Santos, G. S., Dos Santos, A. W., Silva, C. H. S., Barros, J. F., Mendes, T. D. S., \& Góes, M. A. D. O. (2020). Avaliação da mortalidade de uma UTI de Sergipe segundo escore fisiológico agudo simplificado (SAPS 3). Revista de Epidemiologia e Controle de Infecção, 10(1), 1-7. https://doi.org/10.17058/jeic.v1i1.13939

Dessap, A. M., Roche-Campo, F., Launay, J. M., Charles-Nelson, A., Katsahian, S., Brun-Buisson, C., \& Brochard, L. (2015). Delirium and circadian rhythm of melatonin during weaning from mechanical ventilation an ancillary study of a weaning trial. Chest, 148(5), 1231-1241.

Fassini, P., \& Hahn, G. V. (2012). Risk Management in Hospital Unit: Conceptions for Nursing Staff. Rev Enferm UFSM, 2(2), $290-299$.

Françolin, L., Gabriel, C. S., Bernardes, A., De Camargo Silva, A. E. B., De Fatima Paiva Brito, M., \& Machado, J. P. (2015). Patient safety management from the perspective of nurses. Revista Da Escola de Enfermagem, 49(2), 277-283.

Lago, M. S., Faustino, T. N., Mercês, M. C. das, E Silva, D. D. S., Da Cruz Pessoa, L. S., \& Santos Oliveira, M. T. (2020). Delirium e fatores associados em unidades de terapia intensiva: estudo piloto de coorte. Revista Enfermagem Contemporânea, 9(1), 16. https://doi.org/10.17267/2317-3378rec.v9i1.2501

Leão, F. G. de A., Marques, I. D. B., \& Mello, P. M. V. de C. (2019). Validação do índice prognóstico SAPS 3 em pacientes internados na UTI de um hospital terciário de Teresina (PI). Jornal de Ciências Da Saúde Do Hospital Universitário Da Universidade Federal Do Piauí, 1(3), 9. https://doi.org/10.26694/25950290.2018139-197207

Lima, C. S. P., \& Barbosa, S. de F. F. (2015). Ocorrência de eventos adversos como indicadores de qualidade assistencial em unidade de terapia intensiva. Rev. Enferm. UERJ, 23(2), 222-228.

Llapa-Rodríguez, E. O., Oliveira, J. K. A. de, Melo, F. C., Silva, G. G. da, Mattos, M. C. T. de, \& Macieira, V. P. (2019). Inserção de cateter vascular central: adesão a bundle de prevenção de infecção. Revista Brasileira de Enfermagem, 72(3), 774-779.

Lorenzini, E., Santi, J. A. R., \& Báo, A. C. P. (2014). Segurança do paciente: análise dos incidentes notificados em um hospital do sul do Brasil. Revista Gaúcha de Enfermagem, 35(2), 121-127.

Martins, J. B., Dos Santos, A. A., De Macedo Júnior, L. J. J., \& Eberle, C. C. (2019). Avaliação Da Prevalência De Delirium Em Uma Unidade De Terapia Intensiva Pública. Enfermagem Em Foco, 10(3), 76-81. https://doi.org/10.21675/2357-707x.2019.v10.n3.1759

Matioli, K. B. B., Moraes Filho, I. M. de, Sousa, T. V. de, Pereira, M. C., Silva, R. M. da, Sá, E. S. de, \& Oliveira, M. L. C. de. (2021). Delirium: Prevalência E Fatores Associados Ao Pós-Operatório De Cirurgia Cardiovascular Em Idosos. Revista Baiana de Enfermagem35, (April). https://doi.org/10.18471/rbe.v35.42203

Mesa, P., Previgliano, I. J., Altez, S., Favretto, S., Orellano, M., Lecor, C., Soca, A., \& Wesley, E. (2017). Delirium in a Latin American intensive care unit. A prospective cohort study of mechanically ventilated patients. Revista Brasileira de Terapia Intensiva, 29(3), 337-345. https://doi.org/10.5935/0103-

507X.20170058 
Research, Society and Development, v. 10, n. 11, e165101118378, 2021

(CC BY 4.0) | ISSN 2525-3409 | DOI: http://dx.doi.org/10.33448/rsd-v10i11.18378

Minuzzi, A. P., Salum, N. C., Locks, M. O. H., Amante, L. N., \& Matos, E. (2016). Contributions of healthcare staff to promote patient safety in intensive care. Escola Anna Nery - Revista de Enfermagem, 20(1), 121-129.

Novaretti, M. C. Z., Santos, E. de V., Quitério, L. M., \& Daud-Gallotti, R. M. (2014). Nursing workload and occurrence of incidents and adverse events in ICU patients. Revista Brasileira de Enfermagem, 67(5), 692-699.

Nunes, F., Barros, L., Azevedo, R., \& Paiva, S. (2014). Patient safety: how nursing is contributing to the issue? Revista de Pesquisa: Cuidado é Fundamental Online, 6(2), 841-847.

Oliveira, A. C. D., Garcia, P. C., \& Nogueira, L. D. S. (2016). Carga de trabalho de enfermagem e ocorrência de eventos adversos na terapiaintensiva: revisão sistemática. Revista da Escola de Enfermagem da USP, 50, 0683-0694.

Oliveira, M. L. L., \& Nunes, R. D. (2015). Bundles de prevenção da pneumonia associada à ventilação mecânica em unidade de terapia intensiva. Revista Amazônia Science \& Health, 3(2), 36-43. http://ojs.unirg.edu.br/index.php/2/article/view/906

Ortega, D. B., D’Innocenzo, M., Silva, L. M. G. da, \& Bohomol, E. (2017). Análise de eventos adversos em pacientes internados em unidade de terapia intensiva. Acta Paulista de Enfermagem, 30(2), 168-173.

Roque, K. E., Tonini, T., \& Melo, E. C. P. (2016). Eventos adversos na unidade de terapia intensiva: impacto na mortalidade e no tempo de internação em um estudo prospectivo. Cadernos de Saúde Pública, 32.

Silva Junior, J. M., Malbouisson, L. M. S., Nuevo, H. L., Barbosa, L. G. T., Marubayashi, L. Y., Teixeira, I. C., Nassar Junior, A. P., Carmona, M. J. C., Silva, I. F. da, Auler Júnior, J. O. C., \& Rezende, E. (2010). Aplicabilidade do escore fisiológico agudo simplificado (SAPS 3) em hospitais brasileiros. Revista Brasileira de Anestesiologia, 60(1), 20-31. https://doi.org/10.1590/s0034-70942010000100003

Sousa, P., \& Mendes, W. (2019). Segurança do paciente: criando organizações de saúde seguras. (2nd ed., p. 126). Editora FIOCRUZ.

Souza, M. R. N. S., Contarine, L. M., Coutinho Barreto, J. B., Dos Santos, C. M., \& Torres Bertoza, V. P. dos S. (2018). Obstrução Do Cateter De Nutrição Enteral E a Administração De Fármacos Sólidos Na Unidade De Terapia Intensiva Adulto. Biológicas \& Saúde, 8(26), 42-53.

Toffoletto, M. C., Barbosa, R. L., Andolhe, R., Oliveira, E. M. de Janzantte Ducci, A., \& Padilha, K. G. (2016). Fatores relacionados à ocorrência de eventos adversos em pacientes idosos críticos. Revista Brasileira de Enfermagem, 69(6), 1039-1045.

Safety, W. P., \& World Health Organization. (2010). Conceptual framework for the international classification for patient safety version 1.1: final technical report January 2009 (No. WHO/IER/PSP/2010.2). World Health Organization. 